\title{
Supporting scalable video transmission in MANETs through distributed admission control mechanisms
}

\author{
P. A. Chaparro, J. Alcober \\ Telematics Department \\ Technical University of Catalonia \\ 08034 Barcelona, Spain \\ e-mail: \{pedro.alonso.chaparro, \\ jesus.alcober\}@upc.edu
}

\author{
J. Monteiro \\ University of Algarve \\ Faro 8000-810, Portugal \\ e-mail: jmmontei@ualg.pt
}

\author{
C. T. Calafate, J.-C. Cano, P. Manzoni \\ Department of Computer Engineering \\ Technical University of Valencia \\ 46022 Valencia, Spain \\ e-mail: \{calafate, jucano, \\ pmanzoni\}@disca.upv.es
}

\begin{abstract}
Emerging multimedia applications over mobile devices are becoming very popular, especially over infrastructure wireless networks such as cellular and WLANs. However, providing this kind of services over infrastructureless networks like ad hoc networks presents many additional problems. One of these problems is how to share resources fairly among the users involved. In this article we propose a QoS framework supporting scalable video streaming in mobile ad hoc networks based on distributed admission control and video traffic awareness. Our framework promotes fairness between video flows in terms of resource consumption. It also guarantees a significant reduction of the idle times experienced by users during periods of network saturation, thus increasing the video playout time in reception for all users. Using the IEEE 802.11e MAC technology as our basis for traffic differentiation, our framework, called DACMESV (Distributed Admission Control for MANET's - Scalable Video), relies on a periodic probing process to measure the available bandwidth and the end-to-end delay on the path. DACME-SV adopts a cross-layer approach to determine the optimum number of video layers to transmit at any given time, thus avoiding network congestion and guaranteeing an acceptable video quality at the destination. Experimental results show that idle time periods are substantially decreased, while exhibiting a good overall performance in terms of throughput and delay.
\end{abstract}

Keywords-scalable video, distributed admission control, ad hoc networks

\section{INTRODUCTION}

Wireless communications are becoming increasingly important in our everyday lives. Services provided by companies with the goal of increasing their clients and revenues have evolved from short message services to applications such as video streaming and video-conference. The particular feature of wireless networks is that they must satisfy the users' freedom demands, allowing them to be online wherever they are. Among all the different kinds of wireless networks available nowadays, Mobile Ad Hoc Networks (MANETs) [1] are expected to be widely deployed in the future. MANETs are self-organizing wireless networks composed by autonomous mobile nodes and requiring no fixed infrastructure. These networks are characterized by a dynamic topology due to node mobility and variable transmission ranges, where each node must also act as an independent router. Offering real time services over these networks is a real challenge due to their infrastructure-less features, which require more complex routing protocols to counteract the effects of link breakage caused by mobility, channel fading or nodes dynamically joining and leaving the network.

Supporting real-time video on MANETs is a complex task. In fact, the solutions adopted by cellular or Wi-Fi infrastructure networks are not suitable for MANETs since the video packets delivery effectiveness is highly dependent on the network's state. Therefore, new techniques must be adopted to offer acceptable QoS levels to video traffic.

In this work we propose DACME-SV, an extension to the DACME architecture [2] supporting scalable video transmission over MANET environments. DACME$\mathrm{SV}$ is a cross-layer solution between DACME and the H.264/SVC [3] codec. With H.264/SVC the video stream is divided into different layers that provide different degrees of quality. The more layers are received at the destination, the better the video quality. DACME-SV is based on an admission control scheme that gains awareness of the different data flows related to video layers of a same session. Simulation experiments show that DACMESV allows to improve the performance and stability of real-time videoconferencing sessions. With respect to the DACME scheme, DACME-SV is able to reduce by up to $25 \%$ the idle communication times of video sources. Moreover, channel usage as well as the fairness among users requiring QoS support are also improved by adopting the proposed framework.

The rest of this paper is organized as follows: Section II offers an overview of some works related with scalable video support on mobile ad hoc networks. In Section III we describe the original DACME QoS Framework. Section IV describes the scalable extensions to the H.264 standard. The DACME-SV proposal, which supports scalable video through strong cross-layer interactions, is presented in Section V. In Section VI we present our experimental results. Finally Section VII concludes the paper, including some guidelines for future work.

\section{RELATED WORKS}

In the past, several studies have addressed the problem of video transmission in mobile ad hoc network environments. Some of these solutions have been analyzed in [4], 
where the authors present an overview of the state-of-theart in efficient and resilient video coding and protection solutions, including the network routing and transport protocols, link layer QoS support enhancements, as well as cross-layer design for media delivery over multi-hop environments. Another type of solution adopted for video transmission in MANETs is based on multicast. Video multicasting is an efficient bandwidth-saving strategy for many video applications that involve the delivery of a same video to a group of receivers sharing the same multicast address. In [5], a new multicast routing protocol is proposed supporting the transmission of two different streams over two highly-disjoint trees. Their results show that performance is improved over solutions with only one tree; nevertheless, compared to other proposals, the technique used is not very efficient.

Presently, most of the video delivery solutions for ad hoc networks do not take into consideration recent developments in terms of scalable video codecs [3]. Scalable Video Coding techniques provide scalable video streams, which are composed of a base layer and one or more enhancement layers. Enhancement layers may enhance the temporal resolution (i.e. the frame rate), the spatial resolution (i.e. image size), or the signal-to-noise ratio resolution (i.e. SNR) of the content of lower layers. These techniques can also offer advantages to error prone environments such as wireless networks, and even more to ad hoc networks. A more recent work dealing with SVC video delivery is [6]; it presents a solution that improves the playback time using multiples sources for the same video; such solution takes advantage of network coding techniques, as well as of the use of scalable video coding provided by the scalable extensions to H.264; however, this proposal adds complexity to the system since network coding causes significant processing delays on nodes.

To the best of our knowledge, there is currently no proposal of a QoS framework for MANETs offering scalable video awareness at the admission control layer.

\section{ORIGINAL DACME QOS FRAMEWORK}

DACME [2] is a distributed admission control system for MANETs that efficiently handles multi-constrained real-time flows by periodically assessing end-to-end conditions on the path. With DACME, the agent at the video source node performs path probing to obtain different QoS measurements of the end-to-end path, thus assuring that the transmission of video data is made under good conditions. The DACME agent at the destination replies to probes, thus offering the source feedback about path conditions. Also, DACME improves its performance by exploiting a cross-layer optimization between the network layer and DACME agents. This cross-layer relation allows DACME agents to be aware of routing protocol messages, avoiding to send information over paths possibly unavailable; besides, it assesses the new QoS state as soon as a new path is discovered. At the MAC layer it takes advantage of the IEEE 802.11e standard [7] to provide prioritized medium access.

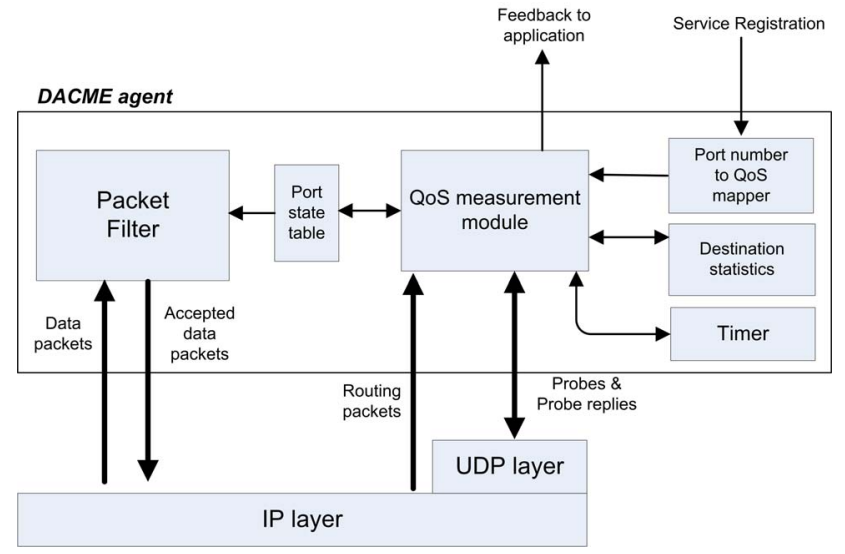

Figure 1. Functional block diagram of the DACME agent.

The main elements of DACME are a QoS module and a packet filter (see Figure 1). The first one is responsible for assessing QoS parameters on an end-to-end path, while the packet filter blocks all traffic which is not accepted into the MANET according to these end-to-end measurements. An application that wishes to benefit from DACME must register itself with the DACME agent, indicating the desired destination UDP ports, along with a QoS specification (Qspec) stating the requested bandwidth, delay, jitter. If any among these values does not meet the application's requirements, DACME may optionally notify this event to the application.

Once registration is successfully completed, the QoS measurement module is activated to periodically perform path probing between source and destination. The destination, after receiving the last probe packet, will send a probe reply back to the source. The QoS measurement module, upon receiving these probe replies, will update the state of the path using per-connection bandwidth, delay and jitter flags. Once enough information is gathered it checks all the registered connections towards that destination, and then decides whether a connection should be accepted, preserved or rejected, updating a port state table accordingly. QoS support becomes effective when the Packet Filter module, according to the Port state table, interacts with the IP layer by configuring the TOS packet header of packets pertaining to accepted data flows. The IEEE 802.11e MAC must then map the service type defined in the IP TOS packet header field to one of the four MAC Access Categories that it makes available.

In [2] we have shown that DACME offers a stable performance when combined with both single and multipath routing protocols. Nevertheless, we found that there was still room for enhancements in terms of reducing on/off states and improving fairness among users. The most straightforward way to achieve these goals is to dynamically adjust the generated data rate so that all active flows benefit from a fair share of the bandwidth. However, performing such tuning at the video codec may be a complex process, especially when the spectrum of possibilities (understood as possible bitrate values) is very wide. The 
use of scalable video emerges as a solution that allows to simplify the problem by offering a pre-determined number of layers with known characteristics. Thus, we avoid continuously updating the codec parameters. More important, the range of data rates offered by scalable video solutions allows combining spatial and temporal resolution with quality.

\section{H.264 ScAlable Video EXTENSION}

In terms of video coding, the Joint Video Team (JVT) from ISO/IEC MPEG and the ITU-T VCEG developed a scalable video coding (SVC) amendment [8] for the H.264/AVC standard [9] that augments the original encoder's functionality to generate several layers of quality. Accordingly, SVC provides scalable video streams [10][11] which are composed of a base layer and one or more enhancement layers. Enhancement layers may enhance the temporal resolution (i.e. the frame rate), the spatial resolution (i.e. image size), or the signal-to-noise ratio resolution (i.e. SNR) of the content represented by the lower layers.

By using the SVC scalability layers, video flows with different levels of quality could be transmitted efficiently over both wired and wireless networks, allowing seamless adaptation to available bandwidth and to the characteristics of the terminal.

In terms of rate adaptation, although SVC enables a wide range of scalability options, transmitting at maximum quality and merely relying on the network to perform rate adaptation according to its degree of congestion or available bandwidth is not acceptable; in fact, in environments such as MANETs, this approach has serious performance consequences. Such solution would limit the number of video streams or devices that simultaneously receive data. It is therefore important to define other mechanisms that can deal with coarse grain rate adjustments for SVC.

The network layer support for scalable video streams, especially by IP layer QoS mechanisms, should be considered as an important complement to rate adaptation techniques since it allows performing a fine tuning between transmission rate and available bandwidth.

\section{A. H.264 SVC Structure}

We now present an overview of the encoding structure of an SVC bit stream. The SVC bit stream may be composed of multiple spatial, temporal, and SNR layers of combined scalability.

Spatial scalability provides support for several display resolutions (e.g. 4CIF, CIF or QCIF) [10], and is implemented by decomposing the original video into a spatial pyramid.

Temporal scalability is a technique that allows supporting multiple frame rates. In SVC, temporal scalability is implemented by using hierarchical prediction structures. A motion-compensated temporal filtering (MCTF) structure [11] can also be used as a preprocessing tool to achieve better coding efficiency.

Quality (or SNR) scalability relies on coarse-grain quality scalable (CGS) or medium-grain quality scalable

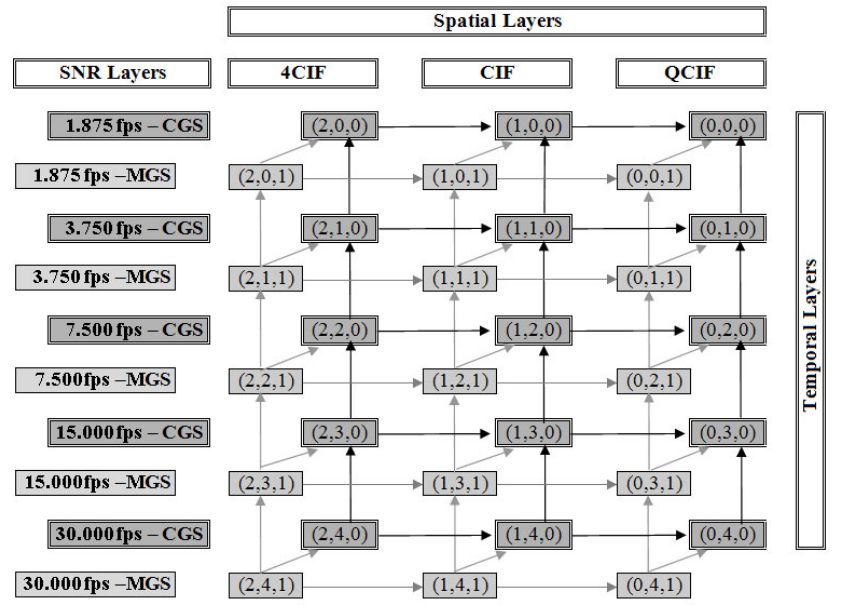

Figure 2. Example of an interdependency structure in a SVC encoded video with three spatial layers, four temporal layers and one MGS layer, where arrows represent NAL unit dependencies.

(MGS) [11]. CGS encodes the transform coefficients in a non-scalable way. In MGS, refinement coefficients can be split in several fragments [12], but cannot be truncated at any arbitrary point, which increases the granularity of rate adaptation options. In this work we use MGS for SNR scalability.

SVC layers are identified using a triple ID, consisting of the Dependency ID, Temporal ID and Quality (i.e. SNR) ID, which is referred as tuple (D,T,Q). For instance, a base layer Network Abstraction Layer (NAL)[9] unit of the lowest temporal resolution and SNR scalability should be identified as $(\mathrm{D}, \mathrm{T}, \mathrm{Q})=(0,0,0)$.

SVC layers can be highly interdependent on each other, which means that the loss of a Network Abstraction Layer (NAL) unit of a certain video layer may cause a severe reduction of quality or even prevent the correct decoding of other layers. For instance, if a 4CIF video sequence with $30 \mathrm{fps}$ is encoded in SVC with the structure represented in Figure 2 and, inside a certain Group of Pictures (GOP), a NAL unit $(1,2,0)$ is lost, then all NAL units that depend on it couldn't be decoded even if they are correctly received. Based on this example, even if the NAL unit $(2,2,0)$ of the same GOP is received, it couldn't be used for decoding and the same happens with other interdependent NAL units.

Regarding the structure presented in Figure 2, we could argue that packet losses affecting the base layer - identified with $\left(0,{ }^{*}, 0\right)$ - have a dramatic impact on the upper layers. Thus, QoS schemes should act to reduce losses to a minimum, thereby avoiding cascading information disruption problems that could render most video data useless.

\section{DACME-SV: SCALABLE VIDEO EXTENSIONS TO DACME}

The fact that the original DACME framework was only able to handle independent flows impeded a joint treatment of correlated flows, which occurs for example when several data streams pertain to a single source of video. DACME-SV is an extension to the original framework endowing it with scalable video awareness. The goals 
pursued with DACME-SV are: (i) to improve channel utilization in the presence of several video sessions; (ii) to reduce idle times within a same session, and (iii) to improve resource utilization fairness among users.

For a joint treatment of video layers to be possible at the admission control layer, the QoS specification defined by the application must include a structure containing the average bandwidth consumed by each video layer $\left(b w_{k}\right)$, as well as the corresponding source ports to keep track of them. Upon receiving this specifications, DACME-SV sources acquire end-to-end path information in terms of bandwidth availability to decide how many video layers can be transmitted over the network without congesting it, thus guaranteeing that traffic will not exceed the network's capacity; if the path's characteristics improve, or if, on the contrary, they become worse, DACME-SV will automatically adapt to such network variability.

DACME-SV improves its performance by exploiting a cross-layer optimization between the network layer and DACME-SV agents. This cross-layer interaction allows DACME-SV agents to be aware of routing protocol messages, avoiding sending information over paths that are possibly unavailable; besides, it assesses the QoS characteristics of a new path as soon as it is discovered.

Next we provide some important details concerning DACME-SV functioning.

\section{A. Bandwidth probing}

The end-to-end bandwidth probing process of DACME$\mathrm{SV}$ consists of sending probe packets to the destination periodically. In our experiments we have set the Inter-Probe Time (IPT) to 3 seconds, which offers a responsiveness similar to that provided by, e.g., the AODV [13] routing protocol. This allows DACME-SV to react quickly if the network becomes suddenly congested.

Each bandwidth probe is composed of $n$ packets generated back-to-back. When all the packets from a probe $i$ arrive to the destination node (or only a subset, $N_{i}$, if the timeout is triggered), the DACME-SV agent at the destination will measure the Average Inter-arrival Time $\left(A I T_{i}\right)$ of incoming probe packets:

$$
A I T_{i}=\frac{\triangle t_{r e c_{i}}}{N_{i}-1}(s)
$$

and it will then calculate the available bandwidth by doing:

$$
B M_{i}=\frac{8 \cdot p}{A I T_{i}}(\text { bit } / s)
$$

where $p$ is the packet size used by the application (on average). This bandwidth measurement (BM) is then sent back to the source. The DACME-SV source agent, upon receiving probe reply packets, will collect the $B M$ values sent by the destination agent to be able to reach a decision on how many video layers should be admitted at that time.

Additionally, DACME-SV takes advantage of interactions with the routing protocols, benefiting from their routing layer information to avoid sending probe packets when no path is available. In our case, when relying on AODV for transmission, DACME-SV achieves optimum performance by re-assessing the end-to-end QoS conditions as soon as a routing RREP message from the destination of a QoS flow is received. This way the admission control mechanism is able to react earlier if the new path can not meet the QoS requirements.

\section{B. Improving the accuracy of bandwidth measurements}

MANETs based on IEEE 802.11 technology [14] conform a system with memory and, for that reason, any short term measurement values must be corrected in order to accurately reflect long-term values [15]. Thus we must obtain, for all values of end-to-end path congestion $(c)$, an unbiased estimator $-v_{p}(c)$ - for the long term available bandwidth - $B_{p}^{L T}(c)$ - achievable with a certain packet size $p$.

We have tested with different correction functions, and we found that we are able to achieve high degrees of accuracy by merely relying on short-term measurements of both mean and standard deviation:

$$
v_{p}(c)=\alpha \cdot v_{p}(c)+\beta \cdot \sigma_{p}(c)
$$

Notice that $\alpha$ and $\beta$ are parameters whose optimal value can be obtained through regression. Our purpose is finding values for $\alpha$ and $\beta$ that apply to a wide range of path congestion values. This purpose can be met since we find experimentally that expression

$$
\exists \alpha, \beta \epsilon \mathbb{R}: 1-\frac{v_{p}(c)}{B_{p}^{L T}(c)}<\varepsilon, \forall c \epsilon\left[0, C_{\max }\right], \forall_{p} \epsilon\left[p_{\min ,} p_{\max }\right]
$$

is true for very low error values $(\varepsilon)$.

\section{Scalable-Video Aware Admission Control Algorithm}

DACME-SV relies on probing packets to test the bandwidth availability on an end-to-end path. The DACME-SV agent compares the result obtained through this process with a pre-established bandwidth threshold for each video layer. If the bandwidth available is greater than the one required for layer $n$, the agent takes a decision and will change his state to $n$. Afterward, and based on the agent's state, it will transmit all the packets belonging to video layer $n$ and below. Thus, the framework allows adapting the video quality to the current resource availability, which allows nodes to experience fewer time periods without video data (Idle states). Algorithm 1 presents the DACME$\mathrm{SV}$ admission control mechanism supporting multi-layer video awareness.

This algorithm works in the following manner: initially it estimates a confidence interval for the available bandwidth. If that interval contains possible data rates for the aggregated video traffic, it generates additional probes to refine the process (up to the maximum allowed) until the normalized value for the interval becomes less than $\delta$ (which in our experiments we have set to 15\%). Finally, it proceeds to check how many consecutive video layers (starting from the base layer) can be served by the network according to its current conditions.

This way, DACME-SV is able to dynamically regulate the quality of streaming video sessions by transmitting more or less video layers; such a process avoids any 


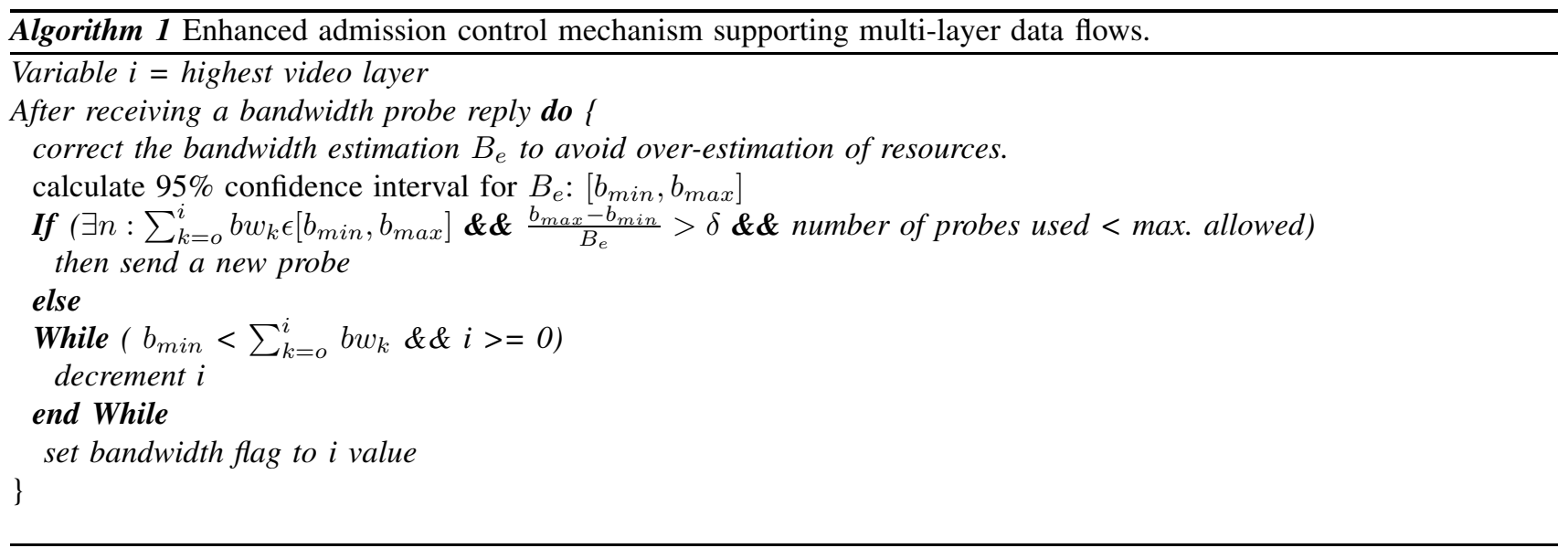

knowledge about the intrinsic details of the codec used. Another important feature of DACME-SV is that it improves fairness among users by avoiding that a few sources monopolize all the available bandwidth. Thus, DACMESV promotes that all video sessions enjoy a similar degree of quality through distributed load balancing between participating nodes.

\section{Vi. Performance Evaluation of DACME-SV}

In this section we analyze the performance of DACMESV using a simulated MANET environment. The simulation platform used is the ns- 2 discrete event simulator [16]. To perform our evaluation we setup a $870 \times 870$ meters scenario with 50 nodes moving according to the random way-point mobility model. All nodes are equipped with an IEEE $802.11 \mathrm{~g}$ interface transmitting at 54Mbit/s. Additionally, all the nodes offer MAC level QoS according to the IEEE 802.11e standard. At the network layer, we employ AODV [13] as the routing protocol. Simulation time is set to 420 seconds; we start video transmission after 100 seconds to make the measurements on a steadystate environment. All measurements were made over a period of 300 seconds and averaged over ten simulation runs, varying the number of video source-destination pairs from 1 to 10 . With respect to video simulation settings, we used real video traffic by adapting the video encoder output to trace formats compatible with ns-2. The video encoder used was the scalable extension to H.264 (JSVM), version 9.10 .

The video sequence used was the well known CREW sequence [17], repeated during 300 seconds. The sequence was captured at 30 frames per second and encoded with a GOP size of 16 at three different resolutions - QCIF, CIF and 4CIF - with two SNR levels for each resolution. Thus, we have a total of six video layers, and the output bitrate varies from $170 \mathrm{kbps}$ (layer 0 alone) to $2.2 \mathrm{Mbps}$ (layers 0 to 5), depending of the number of layers transmitted. The number of layers and their characteristics were selected taking as reference a previous work [18]; the bandwidth associated to the different layers is shown in Table I. These values are aggregated, meaning that to transmit Layer 3 we also have to transmit those layers below it, i.e., 0,1 , and 2 , and so the total bandwidth required would be of $676,8 \mathrm{kbps}$.

In our experiments we also introduce four FTP/TCP sources as background traffic in the best-effort MAC category of IEEE 802.11e (AC_BE) to make the MANET operate under more realistic assumptions. Concerning video traffic, DACME-SV assigns all packets to the Video category of IEEE 802.11e (AC_VI).

\section{A. Throughput, delay and channel utilization results}

We first focus on the experimental results obtained in terms of throughput, delay and channel utilization. Notice that our main goals with DACME-SV are to reduce the idle times of real-time sessions, to increase the fairness among competing users and to improve channel utilization. In terms of average throughput and delay values, these should be maintained similar to those for the standard DACME implementation.

Figure 3 shows the results obtained in terms of throughput. When there is more than a video source active the throughput measured corresponds to the average value among them all; the same applies to delay and channel utilization results. Overall, we observe that the throughput values are quite similar in both systems as intended.

Concerning delay, figure 4 shows that delay values are moderately increased with DACME-SV, although not imposing any restrictions to real-time video traffic (values remain below $100 \mathrm{~ms}$ ).

To better understand the increase perceived in terms of delay, in Figure 5 we show the average network utilization when varying the number of video sources. We can see that Table I

CHARACTERIZATION OF THE DIFFERENT VIDEO LAYERS INVOLVED IN TERMS OF BITRATE AND SPATIAL RESOLUTION.

\begin{tabular}{|c|c|c|c|}
\hline Layer & Aggregated Bit rate (kbps) & Resolution & SNR \\
\hline \hline 0 & 178.5 & QCIF & Low \\
\hline 1 & 190.2 & QCIF & High \\
\hline 2 & 626.5 & CIF & Low \\
\hline 3 & 676.8 & CIF & High \\
\hline 4 & 1959.7 & 4CIF & Low \\
\hline 5 & 2236.0 & 4CIF & High \\
\hline
\end{tabular}




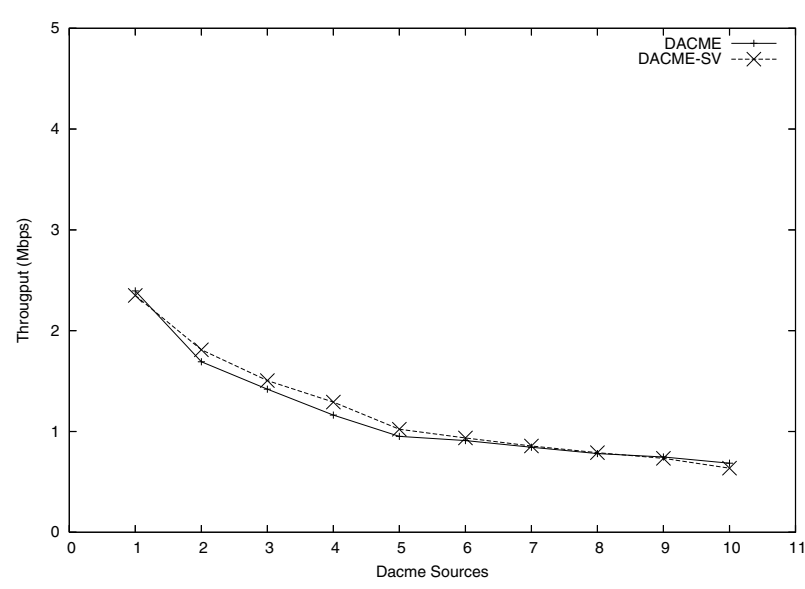

Figure 3. Throughput results for DACME and DACME-SV when increasing the number of video sources.

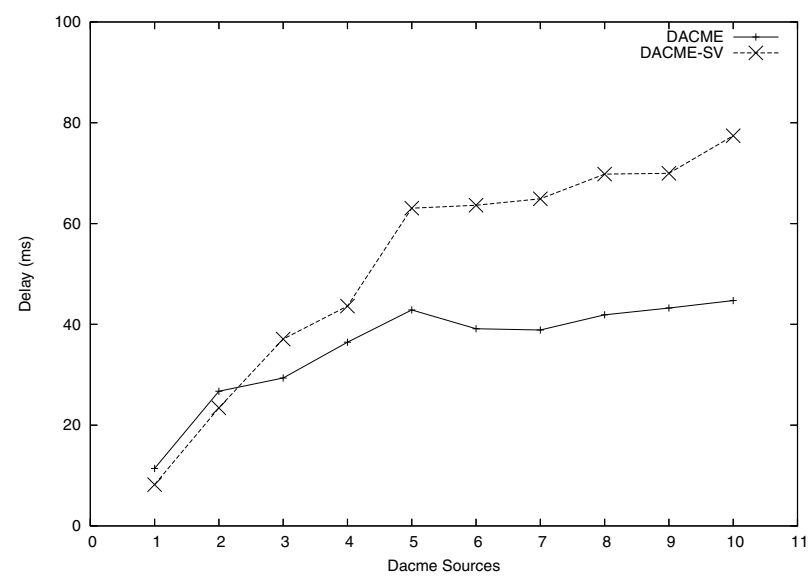

Figure 4. Delay results for DACME and DACME-SV when increasing the number of video sources.

more data is transmitted in the network. However, Figure 3 shows that the differences in terms of throughput between DACME and DACME-SV are minimal. By exploring our experimental results with further detail we found that this increase is mostly related to a higher average number of hops, which results from the desired fairness among users. Now, source-destination pairs separated by a greater hop distance see their chances of transmitting at higher data rates increased, proportionally increasing delay. So, with DACME-SV, greater degrees of congestion are no longer causing connections characterized by high hop counts to be interrupted, which is clearly desirable.

\section{B. Benefits in terms of Idle times}

In this section we present experimental results related to idle times. We call idle time to any period of time during which communication is interrupted due to lack of resources, and, therefore, it is not possible to transmit any video data during such period. Obviously, too many idle time periods will provoke the end-user experience to be poor. Hence, our goal is to reduce the frequency of these interruptions.

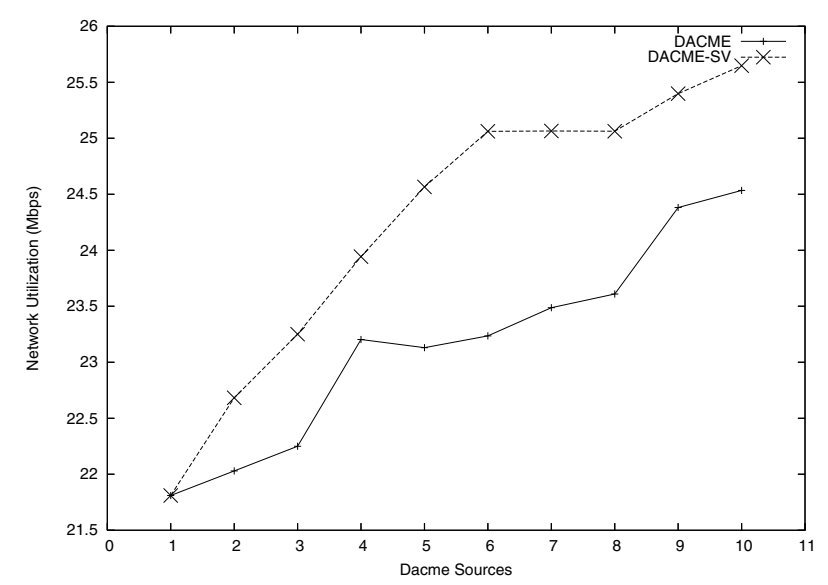

Figure 5. Network utilization results for DACME and DACME-SV when increasing the number of video sources.

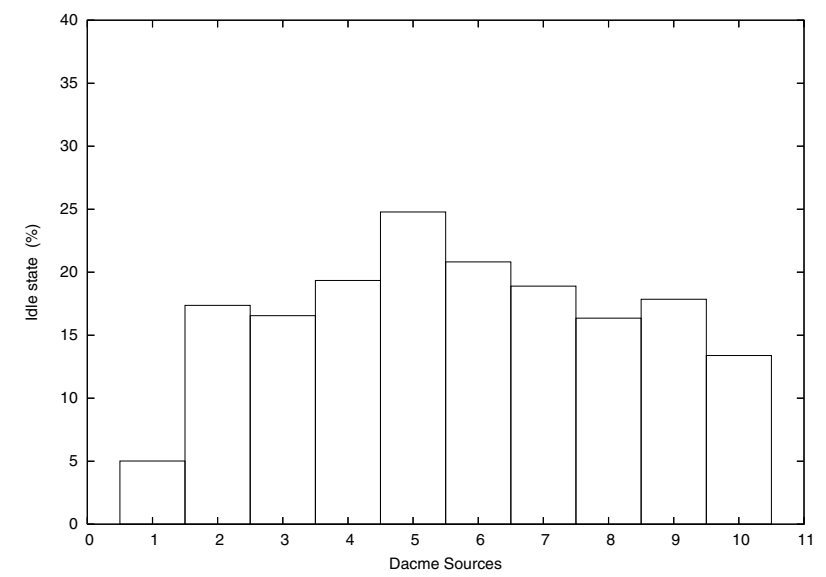

Figure 6. Percentage of idle time improvements for DACME-SV with respect to the original DACME when increasing the number of video sources.

Figure 6 shows the percentage of reduction achieved by DACME-SV with respect to DACME. We can see that DACME-SV is able to clearly reduce idle times from 5\% to $26 \%$, depending of the number of active video sources in the network.

To better understand how DACME-SV is able to reduce idle times without consuming a greater amount of bandwidth, we now detail the degree of video quality perceived by users, which is strictly related to the number of H.264/SVC video layers available for decoding.

Figure 7 presents a comparison between the standard DACME and DACME-SV in terms of video visualization experience as perceived by the end user. In black we represent the percentage of time during which video is not transmitted because the network is unable to support it (due to lack of bandwidth). Notice that the amount of full quality video time (shown in white) is similar for both versions of DACME, being improvements made in terms of reducing idle periods by fully taking advantage of residual bandwidth. As a secondary issue, on the right bar (DACME-SV) we observe that the results for Layer 4 are not available; this is because its bandwidth requirements 


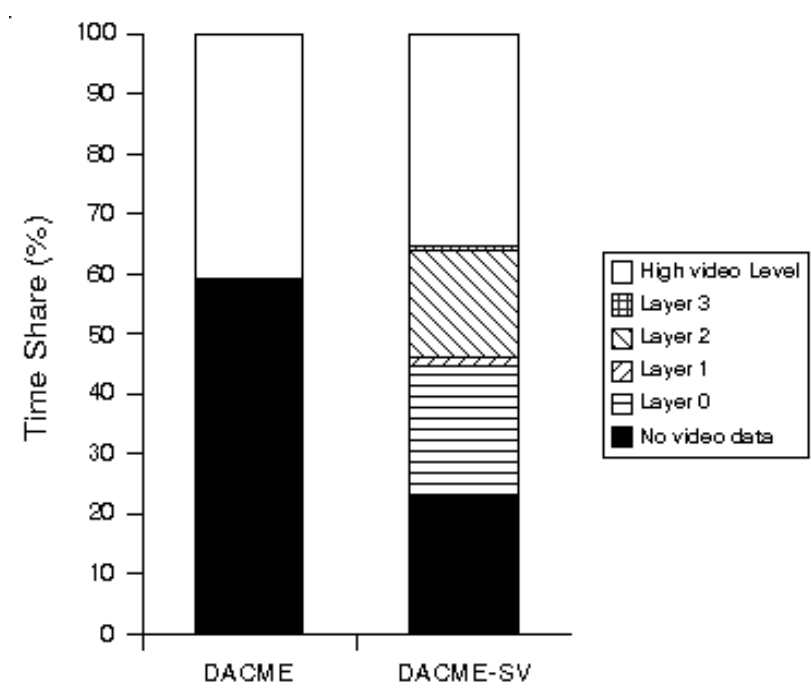

Figure 7. Comparison of Video Levels when simulating with five video sources.

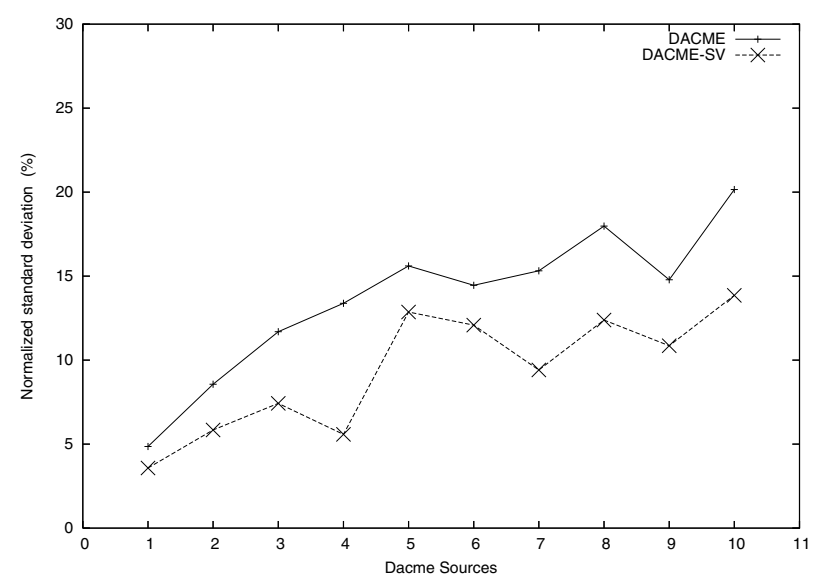

Figure 8. Standard deviation results for idle times with DACME and DACME-SV when increasing the number of video sources.

are very similar to those of layer 5 (High video level). This merely means that, when the available bandwidth in our experiments was high, it was always large enough to accommodate all video layers.

\section{Measuring resource usage fairness}

Up to now our focus was on reducing idle times from a global point of view without compromising network performance. To complete our evaluation we now concentrate on the issue of fairness. Our purpose is to assess if users benefit from a similar resource usage or if, on the contrary, some users experience excellent conditions while others experience very poor ones.

The metric we have chosen was the normalized standard deviation for idle times. Notice that lower values indicate a similar experience by all users, while high values indicate that some users are experiencing fluid communications conditions while others are often experiencing communication disruptions.

Our findings are presented in Figure 8, where we can observe that DACME-SV is able to offer substantial im- provements in terms of user fairness, achieving reductions of up to 8 percent points for the normalized standard deviation values.

Overall, we consider that DACME-SV has successfully met the design goals set, and indeed the greater flexibility offered by the H.264/SVC codec was translated into network-level and user-level improvements.

\section{CONCLUSIONS AND FUTURE WORK}

In this paper we presented DACME-SV, a novel QoS framework to support scalable video transmission over MANETs. We showed the benefits of using this novel solution that combines the flexibility of the H.264/SVC codec with distributed admission control algorithms. The strategy proposed allows to dynamically adjust the video quality according to end-to-end path conditions, thus optimizing available resource usage.

Experimentally we showed that DACME-SV is able to improve global performance by decreasing the frequency of interruptions on video communications, while simultaneously improving fairness among users by favoring similar conditions for QoS flows. Channel utilization was also improved with our strategy.

As future work we plan to introduce further improvements to DACME-SV by also including support for endto-end delay bounds.

\section{ACKNOWLEDGMENTS}

This work was partially supported by the Ministerio de Educación y Ciencia, Spain, under Grants TIN200806441-C02-01 and TSI2007-66637-C02-01, and by the Generalitat Valenciana, Spain, under Grant GV/2009/010.

\section{REFERENCES}

[1] IETF, "MANET Working Group Charter," http://www.ietf.org/html.charters/manet-charter.html.

[2] C.T. Calafate, M.P. Malumbres, J. Oliver, J.C. Cano, and P. Manzoni, "QoS Support in MANETs: a Modular Architecture Based on the IEEE 802.11e Technology," IEEE Transactions on Circuits and Systems for Video Technology, vol. 19, no. 5, pp. 678-692, May 2009.

[3] H. Schwarz, D. Marpe, and T. Wiegand, "Overview of the Scalable Video Coding Extension of the H.264/AVC Standard," IEEE Transactions on Circuits and Systems for Video Technology, vol. 17, no. 9, pp. 1103-1120, September 2007.

[4] Qian Zhang, "Video Delivery over Wireless Multi-hop Networks," International Symposium on Intelligent Signal Processing and Communication Systems (ISPACS 2005), pp. 793-796, December 2005.

[5] CheeOnn Chow and Hi. Ishii, "Video Multicast over Mobile Ad Hoc Networks: Multiple-Tree Multicast Ad Hoc on-Demand Distance Vector Routing Protocol (MTMAODV)," IEEE 18th International Symposium on Personal, Indoor and Mobile Radio Communications (PIMRC 2007), pp. 1-5, September 2007. 
[6] T. Schierl, K. Gaenger, C. Hellge, T. Stockhammer, and T. Wiegand, "SVC-based Multisource Streaming for Robust Video Transmission in Mobile Ad hoc Networks," IEEE Wireless Communications, vol. 13, no. 5, pp. 96-103, October 2006

[7] IEEE 802.11 WG, "802.11e IEEE Standard for Information technology- Telecommunications and information exchange between systems - Local and metropolitan area networks - Specific requirements Part 11: Wireless LAN Medium Access Control (MAC) and Physical Layer (PHY) specifications: Amendment 8: Medium Access Control (MAC) Quality of Service Enhancements," 2005.

[8] ITU-T and ISO/IEC JTC1, JVT-W201, "Joint Draft 10 of SVC Amendment," Joint Video Team (JVT) of ISO-IEC MPEG, April 2007.

[9] Snjezana Rimac-Drlje, Ognjen Nemcic, and Mario Vranjes, "Advance Video Coding for Generic Audiovisual Services," ITU-T Rec. H.264|ISO/IEC IS 14496-10 AVC, vol. 3, September 2005.

[10] H. C. Huang, "Advances in the Scalable Amendment of H.264/AVC,' IEEE Communications Magazine, vol. 45, pp. 68-76, January 2007.

[11] H. Schwarz, D. Marpe, and T. Wiegand, "Overview of the Scalable Video Coding Extension of the H.264/AVC Standard," IEEE Transactions on Circuits and Systems for Video Technology, vol. 17, no. 9, pp. 1103-1120, September 2007.

[12] H. Kirchhoffer, H. Schwarz, and T. Wiegand, "CE1: Simplified FGS," Joint Video Team (JVT) of ISO-IEC MPEG, April 2007.

[13] C. Perkins, E. Belding-Royer, and S. Das, "Ad Hoc Distance Vector (AODV) Routing," RFC3561 , IETF, July 2003.

[14] IEEE 802.11 WG, "International Standard for Information Technology - Telecom. and Information exchange between systems - Local and Metropolitan Area Networks - Specific Requirements - Part 11: Wireless Medium Access Control (MAC) and Physical Layer (PHY) Specifications, ISO/IEC 8802-11:1999(E) IEEE Std. 802.11,” 1999.

[15] Carlos T. Calafate, Pietro Manzoni, and Manuel P. Malumbres, "Supporting soft real-time services in MANETs using distributed admission control and IEEE 802.11e technology," in The 10th IEEE Symposium on Computers and Communications, La Manga del Mar Menor, Cartagena, Spain, June 2005.

[16] K. Fall and K. Varadhan, "ns notes and documents." The VINT Project. UC Berkeley, LBL, USC/ISI, and Xerox PARC, February 2000.

[17] "H.264/SVC Test Sequences (2008, July 27). Institute of Signal Processing, Hannover University, Germany," [Online]. Available: ftp://ftp.tnt.uni-hannover.de/pub/svc/.

[18] Janio M. Monteiro, Carlos T. Calafate, and Mario S. Nunes, "Evaluation of the H.264 Scalable Video Coding in Error Prone IP Networks," IEEE Transactions on Broadcasting, vol. 54, no. 3, pp. 652-659, September 2008. 